\section{Conhecimentos e práticas dos profissionais de saúde sobre a "atenção humanizada ao recém-nascido de baixo peso - método canguru"}

\author{
Health professional's knowledge and \\ practices about "human attention of low \\ birth weight- kangaroo care"
}

Marcia de Abreu e Silva Hennig1 Maria Auxiliadora de Souza Mendes Gomes2 Nicole Oliveira Mota Gianini3

1-3 Instituto Fernandes Figueira - IFF FIOCRUZ/RJ. Av. Rui Barbosa, 716 - Flamengo. Rio de Janeiro, RJ, Brasil. CEP: 22.250-020 Tel: 21 2554.1700 - Fax: 212553.6730. E-mail:mabreu@rio.com.br

\begin{abstract}
Objective: to identify knowledge and practices related to Low Weight Newborn Humane Care - the Kangaroo Method by the medical and nursing staff in public maternity hospitals located in the city of Rio de Janeiro.

Method: a cross cutting descriptive method performed by questionnaire application aiming at the management of eleven public maternity hospitals in the city of Rio de Janeiro, selected for the study, related to humane medical care for low weight newborns - the Kangaroo Method. One hundred and forty eight doctors and nurses working at these hospitals were interviewed. Of these, 116 professionals exclusively working in ICU answered specific questions related to care under the perspective of the Kangaroo Method.

Results: as for the strategies noted to minimize noise and luminosity, 39\% of the professionals related prompt answer to alarms/careful equipment use and $88 \%$ referred to luminosity reduction in at least one period out of 24 hour. As for strategies to reduce pain/discomfort, $34 \%$ reported the use of non-feeding suction and $9 \%$ reported on the use of glucose. Eighty three percent reported clinical data as the main information relayed to parents during the first visit. Physical exam is a procedure where the mother's presence is allowed by $73 \%$ of the respondents. Fifty nine percent reported that clinical stability was the reason of allowing the mothers to hold the baby for the first time, but $30 \%$ will permit that only if the new born is stable with breathing support.

Conclusion: notwithstanding theoretical knowledge on Humane Care, professionals still do not apply it fully in their clinical practice, which is suggestive that the scope of this type of neonatal care is still not totally accepted.
\end{abstract}

Key words Kangaroo method, Healthcare, Low birthweight baby, Humane Care Program during prenatal and birth assistance, Professional practice.

\section{Resumo}

Objetivo: identificar os conhecimentos e as práticas sobre a Atenção Humanizada ao RecémNascido de Baixo Peso - Método Canguru (AHRNBP$M C)$ de profissionais médicos e de enfermagem em maternidades públicas da cidade do Rio de Janeiro.

Método: estudo descritivo transversal realizado através de questionário. Participaram das entrevistas 148 médicos e enfermeiros atuantes nestas unidades. Destes, os 116 profissionais que atuam exclusivamente na unidade intensiva responderam sobre quesitos específicos das práticas assistenciais sobre a perspectiva da AHRNBP-MC.

Resultados: em relação às estratégias apontadas para minimizar ruído e luminosidade, $39 \%$ dos profissionais referiram atender rapidamente aos alarmes/cuidados no uso dos equipamentos e $88 \%$ apontaram a diminuição da luminosidade em pelo menos 1 período em 24h. Como estratégias para dor/desconforto, 34\% referiram usar a sucção nãonutritiva e $9 \%$ citaram utilizar glicose. Oitenta e três porcento relataram serem as informações do quadro clínico as principais informações aos pais na $1^{a}$ visita. O exame físico é o procedimento em que é permitida a presença da mãe para $73 \%$ dos entrevistados. Cinquenta e nove porcento usavam a estabilidade clínica como critério para a ida do bebê ao colo materno pela primeira vez.

Conclusão: apesar do conhecimento teórico sobre a Atenção Humanizada, os profissionais ainda não o utilizam plenamente em sua prática clínica, sugerindo que ainda não está completamente assimilada a abrangência dessa forma de cuidado neonatal.

Palavras-chave Método canguru, Cuidados de saúde, Recém-nascido de baixo peso, Programa de Humanização no Pré-Natal e nascimento, Prática profissional. 


\section{Introdução}

A crescente visibilidade da mortalidade neonatal tem sido um desafio para o sistema de saúde brasileiro. Assim sendo, a busca por medidas para enfrentar os problemas existentes na assistência perinatal, incluindo o atendimento de maior complexidade, passou a integrar a agenda de prioridades das políticas públicas em saúde no Brasil. ${ }^{1}$

O Baixo Peso ao Nascer (BPN), definido como peso abaixo de $2500 \mathrm{~g}$, e a prematuridade são grandes responsáveis pela mortalidade neonatal, representando $69 \%$ de todos os óbitos neonatais, e pelos distúrbios funcionais entre os sobreviventes. ${ }^{2}$

Nos países desenvolvidos a implantação e o impacto das ações desenvolvidas na área perinatal, somadas ao desenvolvimento da neonatologia e às novas tecnologias surgidas nas duas últimas décadas, têm possibilitado a sobrevida de recém-nascidos (RN) cada vez menores. No Brasil, as elevadas taxas de mortalidade neonatal ainda se constituem um desafio para o nosso sistema de saúde. ${ }^{2}$

Ao lado da preocupação inicial com as taxas de sobrevivência dos neonatos de risco, nos últimos anos uma outra questão despertou grande preocupação: as conseqüências advindas das intervenções realizadas no período da hospitalização, por vezes prolongada, desses RN. As atenções se voltaram, então, às seqüelas em seu desenvolvimento, percebendo-se a importância de estudar não só os elementos biológicos, mas também os aspectos psicossociais envolvidos e as conseqüências que o ambiente, neste caso a Unidade Neonatal (UN), poderia trazer para o desenvolvimento e conseqüentemente, para a qualidade de vida desses bebês e de suas famílias.

A preocupação com a separação precoce e prolongada de mãe-bebê e família, os procedimentos dolorosos e/ou desconfortáveis, excessivo manuseio, ruído constante, alimentação enteral, menor prevalência de aleitamento materno, maior exposição a complicações que cursam com graves seqüelas, maior demanda de atenção especial e de alto custo, suscitou várias linhas de pesquisa sobre as formas e conseqüências de intervir neste ambiente. ${ }^{3-8}$

Várias propostas de intervenção, centradas na presença e participação da mãe, surgiram com resultados que chamam a atenção no que se refere ao desenvolvimento global da criança, incluindo as interações sociais e desempenho escolar, a partir da década de 80.9,10

No Brasil, a partir do final da década de 90, esta preocupação traduziu-se na "Atenção Humanizada ao Recém-Nascido de Baixo Peso - Método
Canguru" (AHRNBP-MC) elaborada e implementada pelo Ministério da Saúde (MS), através de norma, protocolos e de um amplo processo de capacitação nas diferentes regiões do país. ${ }^{11}$

A AHRNBP-MC se caracteriza principalmente pela mudança na forma do cuidado neonatal baseada em quatro fundamentos básicos: 11,12

- acolhimento do bebê e sua família;

- respeito às singularidades (cuidado individualizado);

- promoção do contato pele-a-pele o mais precoce possível;

- envolvimento da mãe nos cuidados com o bebê.

O método brasileiro caracteriza-se pelo desenvolvimento em três etapas, interligadas, onde o sucesso de uma etapa depende do adequado trabalho realizado na etapa anterior. 13

$\mathrm{Na}$ primeira etapa, após a identificação da gestante com risco de ter um bebê de baixo peso, essa recebe orientações específicas sobre os cuidados a serem tomados. No caso do recémnascido necessitar permanecer na unidade de terapia intensiva neonatal e/ou de cuidados intermediários, especial atenção é dada para estimular a entrada dos pais na unidade, orientar sobre as condições clínicas da criança, oferecer suporte e estímulo para o contato pele-a-pele com o bebê. É trabalhado também o estímulo à lactação e à participação dos familiares nos cuidados com a criança.

A segunda etapa prevê o bebê em situação clínica estável, sua mãe segura e orientada nos cuidados com seu filho, na enfermaria de alojamento conjunto, mantendo a posição canguru pelo período que ambos (mãe e bebê) acharem seguro e conveniente.

A terceira etapa baseia-se no acompanhamento ambulatorial após a alta, com orientação e acompanhamentos especializados.

Para essa abordagem do cuidado neonatal, a readequação das rotinas foi acompanhada pelos desafios inerentes à incorporação de novas práticas, da mesma forma como acontece em outros aspectos assistenciais. Vale destacar que o uso criterioso de antimicrobianos e a nutrição enteral precoce (trófica), práticas já consagradas pelas evidências científicas, ainda não estão plenamente incorporados no cotidiano das Unidades Neonatais (UN). 14-17

Em nosso meio, potencializando as dificuldades na incorporação de novas práticas, encontramos a carência de leitos intermediários e de terapia intensiva, dificuldades na estrutura dos leitos existentes e superlotação nestas unidades, características que estão bem documentadas no setor público brasileiro. 1,11 
Entendendo que as propostas trazidas pela AHRNBP-MC buscam a revisão das práticas e rotinas assistenciais, esse artigo visa identificar os conhecimentos e as práticas sobre a "Atenção Humanizada ao Recém-Nascido de Baixo Peso Método Canguru" de profissionais médicos e de enfermagem em maternidades públicas da cidade do Rio de Janeiro.

\section{Método}

Estudo descritivo transversal, no campo da pesquisa avaliativa, sobre os conhecimentos e práticas dos profissionais de saúde sobre a "Atenção Humanizada ao Recém-Nascido de Baixo Peso - Método Canguru" em 11 maternidades públicas da cidade do Rio de Janeiro, que participaram com pelo menos três profissionais no Curso de Capacitação do Ministério da Saúde, no período de 2000-2002.

Partindo das rotinas e protocolos definidos na norma e no manual de capacitação da AHRNBP$\mathrm{MC}$, foram selecionados indicadores objetivando conhecer sua incorporação pelas Unidades Neonatais capacitadas.

As maternidades participantes do estudo foram:

- Instituto Fernandes Figueira - FIOCRUZ

- Hospital Geral de Bonsucesso

- Hospital Estadual Rocha Faria

- Maternidade Escola da Universidade Federal do Rio de Janeiro - UFRJ

- Hospital Pedro Ernesto da Universidade

Estadual do Rio de Janeiro - UERJ

- Hospital Maternidade Oswaldo Nazareth (Praça XV)

- Instituto Municipal da Mulher Fernando Magalhães - IMMFM

- Hospital Maternidade Carmela Dutra

- Unidade Integrada de Saúde Herculano Pinheiro - UISHP

- Hospital Maternidade Alexander Fleming

- Maternidade Leila Diniz

As informações foram obtidas a partir dos dados fornecidos pelas unidades, e de questionários aplicados às chefias e rotinas médica e de enfermagem e plantonistas.

$\mathrm{O}$ agendamento das entrevistas foi realizado previamente, por contato telefônico com os chefes de serviço das unidades, com uma breve apresentação do trabalho e do objetivo do estudo.

Os questionários foram aplicados durante a visita da pesquisadora. Em razão da disponibilidade de horário dos entrevistados, no momento da primeira visita e pelo organograma da unidade, houve neces- sidade de retorno às maternidades buscando abranger a totalidade das chefias e profissionais da rotina de cada serviço.

Foram aplicados dois tipos de questionários: um para as chefias médica e de enfermagem e outro para profissionais da rotina/diaristas e plantões. As questões foram referentes às mesmas variáveis, porém apresentadas com diferentes formulações dependendo da inserção do entrevistado.

Em relação às chefias, investigou-se especificamente as orientações que são dadas para o conjunto da equipe frente às normas de cuidado na perspectiva da atenção humanizada.

Para os profissionais da rotina e plantões as questões foram formuladas em dois aspectos: 1) que orientações receberam e 2) quais eram as suas práticas nas situações específicas que foram selecionadas para a entrevista.

Os indicadores referentes aos elementos da estrutura e das rotinas do cuidado neonatal abrangeram:

a. Número de profissionais treinados no Curso Teórico-Prático em atividade na Unidade Neonatal/Enfermaria Canguru;

b. Ocorrência de estratégias locais de treinamento/disseminação das rotinas preconizadas;

c. Horários para presença dos pais;

d. Direito de visita de avós;

e. Existência de práticas facilitadoras para presença da mãe;

f. Existência de Enfermaria Canguru;

g. Número de leitos na Enfermaria Canguru;

h. Rotinas clínicas para início da segunda etapa e para seguimento ambulatorial ( $3^{\mathrm{a}}$ etapa).

Para os profissionais, os indicadores foram referentes a:

a. Horários para presença dos pais;

b. Direito de visita de avós;

c. Existência de práticas facilitadoras para presença da mãe;

d. Estímulo/permanência no contato pele-a-pele;

e. Identificação de estratégias para adequação do meio ambiente em relação a ruídos e

estímulos visuais;

f. Estratégias ante condutas dolorosas e/ou causadoras de desconforto no RN.

Este estudo foi elaborado segundo as diretrizes e normas regulamentadoras de pesquisa envolvendo seres humanos - Resolução 196/96 do Conselho Nacional de Saúde, sendo submetido ao Comitê de Ética em Pesquisa do Instituto Fernandes Figueira FIOCRUZ. Foram solicitadas autorizações das Direções das Maternidades selecionadas para o estudo, assim como foi solicitado o consentimento 
de todos os profissionais entrevistados através do Termo de Consentimento Livre e Esclarecido.

\section{Resultados e Discussão}

As 11 maternidades foram responsáveis pelo atendimento de 4.727 prematuros nascidos na cidade do Rio de Janeiro em 2003, dos quais 968 com peso inferior a $1500 \mathrm{~g}$. Assim sendo, as rotinas, os conhecimentos e práticas dos profissionais que cuidam desses recém-natos aqui apresentados permitem mapear a forma de cuidado neonatal que atinge $74 \%$ dos prematuros e $67 \%$ dos muito baixo peso (MBPN) atendidos no Sistema Único de Saúde (SUS), no município do Rio de Janeiro, segundo dados do Sistema de Informação sobre Nascidos Vivos - SINASC. 18

Um ponto interessante a ser destacado foi notar que a grande maioria dos profissionais não identificava o método pelo seu nome - Atenção Humanizada ao Recém-Nascido de Baixo-Peso - Método Canguru (AHRNBP-MC), reconhecendo-o apenas ao ouvirem a palavra canguru.

A AHRNBP-MC no Brasil difere bastante da idealizada e implementada em seu país de origem, a Colômbia. De método canguru ou mãe canguru, a proposta brasileira como o próprio nome o diz, é muito mais abrangente, fundamentando-se num contexto de assistência neonatal mais amplo, baseado em cuidados individualizados, intervenção centrada na família, contato pele-a-pele precoce como facilitador do vínculo entre pais e bebê, intervenções no meio ambiente e na assistência neonatal prestada através de seus profissionais, objeto deste estudo. Entretanto a "marca" canguru é muito forte e as respostas dos profissionais sugerem que ainda não está completamente assimilada essa abrangência da proposta brasileira.

Dentre as unidades estudadas, 7 dispõem de estrutura física diferenciada para internação conjunta mãe-bebê, correspondendo à segunda etapa da AHRNBP-MC (Enfermaria Canguru). A literatura é vasta19-25 sobre a importância do contato mãe-bebê, o mais precoce possível, para a formação do vínculo parental. É necessário oferecer à mãe um espaço físico que permita não só um maior e constante contato físico, mas também que possibilite adquirir gradualmente a confiança em sua capacidade de cuidar e proteger seu filho.

No que se refere a outras estratégias de humanização da atenção ao parto e nascimento, encontramos em 7 das 11 unidades a possibilidade de acompanhante no pré-parto e sala de parto, sendo que 5 maternidades já contam com pré-parto individualizado. Três maternidades relataram trabalhar com suporte específico para a gestante no trabalho de parto/parto. Estes são indícios suficientes de que a revisão das rotinas de atenção ao parto e nascimento está em curso, o que vem ao encontro das propostas da OMS e do MS1. Entretanto, os desafios ainda são muitos. Um exemplo claro é a proporção de partos com acompanhante ter sido de $36 \%$.

Mesmo que apenas na resposta ao questionário, encontramos referência a existência de estratégias para o preparo de gestantes de risco, em 3 das unidades pesquisadas. Vale lembrar que para a própria norma do MS, o início da atenção adequada ao RN antecede ao período do nascimento, quando, uma vez identificada a gestação de risco, essa gestante deve ser encaminhada para unidades de referência de risco e serem oferecidas informações sobre cuidados médicos específicos e humanizados. 26

Para instrumentalizar os profissionais na AHRNBP-MC, o MS elaborou um Programa de Disseminação da Atenção Humanizada, realizado através de cursos de capacitação com a duração de 40 horas, conduzidas, consecutivamente, em uma semana de trabalho, com aulas expositivas, práticas clínicas, discussões, oficinas realizadas pelo grupo de consultores que elaborou o manual técnico, base da norma da AHRNBP-MC. Esses cursos são realizados por todo o país, através de centros de referência, sendo responsável, no Rio de Janeiro, a SMSRJ.18 Além dessa capacitação, estratégias locais de capacitação e reciclagem fazem parte do programa, com cursos variando de 06 a 20 horas, de acordo com a disponibilidade de cada unidade.

Em nosso estudo, destacamos como positivo o fato de $87 \%$ dos profissionais treinados no curso de 40 horas pelo centro de referência, ainda permanecerem em atividade na unidade. Também identificamos 5 maternidades que ofereceram treinamento local, capacitando 154 profissionais. Dentro dessa perspectiva, foi interessante observar que grande parte dos profissionais vincula a AHRNBP$\mathrm{MC}$ apenas à posição canguru (manter o $\mathrm{RN}$ de baixo peso, ligeiramente vestido, em decúbito prono, na posição vertical, contra o peito do adulto) e identifica a atenção humanizada ao momento da redução de luz do ambiente: "Aqui diminuímos a luz quando acaba a visita... nós chamamos de hora da humanização!"

Quando se identificou elementos da estrutura e das rotinas do cuidado neonatal referentes às estratégias ambientais e sua adequação à Norma, em relação aos acessos de mãe e pai às unidades, uma 
situação bastante peculiar foi evidenciada. Quando se perguntou havia acesso livre dos pais para verem seu bebê, a quase totalidade dos profissionais respondeu que sim, com uma ressalva: a necessidade de estabelecimento de horário, em algumas unidades, para a entrada do pai (4 unidades com acesso livre) ou da mãe (6 unidades com acesso livre), nas situações em que ela não estivesse internada com o bebê, por questões de segurança pública (unidades situadas em área de risco).

Em relação ao direito de visita dos avós, ainda na $1^{\mathrm{a}}$ etapa (Unidade de Terapia Intensiva Neonatal UTIN), esta parece ser uma prática já incorporada pelas unidades (10 em 11 unidades), o que ainda não ocorre em relação ao direito de visita de outros irmãos do bebê, alguém escolhido a critério da mãe (6 em 11 unidades). De acordo com a AHRNBP-MC, a idéia principal que norteia o conceito de família ampliada é o reconhecimento de que um bebê sozinho não existe e que zelar pela preservação dos vínculos afetivos familiares é prevenir a saúde de todos os integrantes desses grupos e do bebê.12,22,26,27

Observou-se que $100 \%$ das unidades estudadas ofereceram estratégias para permanência da mãe durante a internação do RN, facilitando o estabelecimento do vínculo entre a mãe e bebê. A condição da prematuridade associada a não permanência da mãe junto ao bebê pode trazer sérias dificuldades para a formação do vínculo parental, situação amplamente descrita na literatura. $9,19,20,27-31$

A presença de atividades de suporte durante a permanência da mãe é garantida por 7 maternidades. As estratégias utilizadas variam desde acompanhamentos em grupos orientados por equipe interdisciplinar (psicólogos, assistentes sociais, fonoaudiólogos, enfermeiros) até grupos de musicoterapia, atividades artesanais e de leitura. A possibilidade de um espaço onde os conflitos, as dúvidas e as experiências possam ser ouvidas e compartilhadas, contribui para minimizar os efeitos estressantes do período de internação do bebê, assim como contribuir para uma melhor ambientação e permanência da mãe nesse período. 20,32-36

Quanto aos itens que foram prevalentes nas respostas dadas aos questionários no que diz respeito a ruído e luminosidade, entrevistamos 148 profissionais, sendo 22 de chefia, 80 de rotina e 46 plantonistas.

Várias recomendações em relação ao ruído e a luz são descritos na literatura para minimizar os efeitos dos estímulos ambientais excessivos produzidos pelas UTI neonatais sobre o desenvolvimento dos RN.3,6,35 Contudo, em relação ao ruído, freqüientemente esses cuidados são desconsiderados: cerca de $31 \%$ dos entrevistados relataram não dispor de nenhuma estratégia para redução de ruído. Também merece ser ressaltado que nenhuma das 11 Unidades Neonatais contava com tratamento acústico, apesar do grande número de pessoas (profissionais, pacientes, funcionários administrativos, dentre outros) e equipamentos existentes nas unidades.

Os efeitos advindos da longa exposição de um $\mathrm{RN}$ prematuro e/ou de baixo peso a um ambiente ruidoso podem trazer como conseqüência, alto risco de hemorragia intraventricular, intercorrência freqüentemente precursora de uma variedade de desordens do desenvolvimento cerebral. Em razão da imaturidade neurológica e instabilidade fisiológica, esses bebês gravemente doentes são especialmente vulneráveis ao excesso de ruído das UTIN, resultando em danos neurológicos e dificuldades na organização cerebral.3,4

Nesta pesquisa observamos que apesar dos profissionais referirem como ruidosa a unidade onde trabalham, quando se perguntou sobre os cuidados tomados para redução de ruídos, dos 148 profissionais, apenas $39 \%$ apontaram atender rapidamente aos alarmes e cuidados no uso de equipamentos como medida para diminuição sonora. O uso do telefone celular foi bastante criticado, porém apenas $37 \%$ referiram restrição quanto ao seu uso e $30 \%$ disseram reclamar quando o barulho estava tornando-se insuportável.

Grande parte dos entrevistados relatou ser o período da manhã o mais ruidoso por ser o horário das visitas médicas, estando presentes nesse horário, a maioria dos profissionais. Outra observação dos profissionais foi o nível do ruído, que pode variar bastante - de médio para alto e de alto para muito alto, de acordo com a equipe do dia.

Diferentemente do ruído, a diminuição setorial da luminosidade foi relatada por grande parte dos profissionais de chefia e rotina $(88 \%)$ e pela quase totalidade dos plantonistas (95\%). Foi interessante observar que, apesar da grande maioria das unidades usar algum tipo de protetor (lençol, emborrachado, cueiro) como black out nas incubadoras, apenas $20 \%$ apontaram o fato como recurso para redução de luz.

Como estratégia mais freqüentemente utilizada por esses profissionais está o apagar as luzes e deixar apenas uma iluminação central acesa, nos períodos da tarde, após a visita médica e durante a madrugada.

A constante exposição à luz é uma das características de unidades de cuidados intensivos onde a 
necessidade de contar com ambiente muito iluminado facilita a adequada monitorização dos pacientes e permite adequação ao trabalho noturno.

A anatomia ocular do prematuro faz com que retenha maior quantidade de luz em sua retina. Como conseqüência da exposição luminosa constante, o neonato pode apresentar dificuldades para se ajustar ao ciclo dia/noite, podendo levar a alterações dos ciclos circadianos endógenos que podem interferir na consolidação normal do sono ou mesmo à sua privação. A adequada utilização dos ciclos dia/noite permite melhora na sincronização de seus ritmos biológicos, aumento das horas de sono noturno, melhora na eficiência alimentar e ganho ponderal além de possibilitar ao bebê explorar e interagir com o meio e com seus pais. ${ }^{25}$

Na elaboração do questionário, houve a preocupação de incluir outros indicadores significativos das práticas assistenciais na perspectiva da AHRNBPMC, cujo caráter é bastante específico da prática individual de cada profissional. Nestas perguntas, temos uma redução do número de profissionais de rotina que responderam, já que alguns deles têm atividades predominantemente no alojamento conjunto ou de natureza gerencial. Responderam a esses quesitos 116 profissionais.

No que se refere às estratégias para dor/desconforto do $\mathrm{RN}$, recursos como técnicas posturais (17\%), adequação dos procedimentos, ou seja, agrupar/intervalar o manuseio doloroso (10\%) e a utilização de glicose (9\%) são apontados pelos entrevistados. Encontrou-se ainda $17 \%$ que relataram usar fármacos durante a realização de procedimentos sabidamente dolorosos (punção lombar, drenagem torácica, pós-operatórios potencialmente dolorosos, dissecação venosa, dentre outros citados). Nesse contexto, de utilização não tão freqüente dos recursos acima mencionados, verificou-se $34 \%$ dos profissionais indicando a sucção não-nutritiva como paliativo em procedimentos dolorosos e $19 \%$ referindo conversar com o bebê como forma de tranquiilizar ou avisar sobre o procedimento realizado. Existe um lapso entre o conhecimento e a conduta clínica e que isso se deve a falhas na incorporação dos conhecimentos científicos a respeito da presença, do diagnóstico e do tratamento da dor na prática diária dos profissionais de saúde também verificado por Chermont et al. 37

$\mathrm{O}$ reconhecimento de que o recém-nascido sente dor e que é capaz de responder aos estímulos nociceptivos é hoje um consenso na comunidade médica. O estudo do desenvolvimento fetal e do comportamento dos prematuros nos permite conhecer os efeitos que a exposição à dor podem provocar nos sistemas sensoriais do neonato. Sua reduzida capacidade de inibição da dor, associada à sua capacidade de percepção dolorosa pode se refletir negativamente, por meio de alterações orgânicas, fisiológicas e comportamentais, repercutindo no desenvolvimento do SNC e até na interação mãe-bebê. ${ }^{38-42}$

Com relação às principais informações dadas aos pais na primeira visita a UN, um percentual expressivo de profissionais $(83 \%)$ relatou ser o quadro clínico a primeira e principal informação dada aos pais. Essa resposta era sempre seguida de uma observação: "da forma mais simples possível, sem termos técnicos para que eles possam entender o real estado do bebê..." Apesar da preocupação explícita nessa fala, em tornar acessível a informação aos pais, fica clara a dificuldade dos profissionais em ouvir a real demanda dos pais sobre o seu bebê. Lamy ${ }^{35}$, citando Green (1979), descreve os pais de bebês internados em UN como "... simplesmente incapazes de entender o que está acontecendo". Em nosso estudo apenas $9 \%$ informaram de acordo com a demanda dos pais. 19,25,28,42,43

A importância e os benefícios advindos da presença e participação dos pais desde a internação do bebê é inquestionável, portanto julgamos importante observar num mesmo contexto, as respostas ao estímulo dado para a mãe no cuidado com o bebê e nos procedimentos em que é permitido a mãe participar.

Tocar, conversar, estar próximo do bebê o maior tempo possível (maternagem) foram itens respondidos por $81 \%$ dos profissionais em relação ao estímulo no cuidado com o bebê. Porém apesar de bastante reconhecido pela literatura os benefícios que essa maternagem pode trazer como atenuante nos manuseios dolorosos e/ou desconfortáveis, 19,23,30,41 apenas $32 \%$ dos entrevistados citaram procedimentos em que a mãe participava.

Nos cuidados assistenciais (troca de fraldas, sucção não-nutritiva), $23 \%$ estimulam como forma de cuidado e $37 \%$ como forma ativa de participação da mãe nos procedimentos. Essa prática não só reforça a confiança materna no cuidado, como também estimula a responsabilidade parental.19,30 Devemos lembrar que esse enfoque dos profissionais não pode ser desvinculado de uma atenção cuidadosa às possibilidades da mãe em cada momento.

Para $31 \%$ dos entrevistados, o estímulo ao cuidado se dá ao incentivar a ida da mãe ao banco de leite para ordenha de leite e para manutenção da lactação (orientação sobre amamentação). Embora esse estímulo, inegavelmente, contribua para reforçar, na mãe, sua importância na melhora do seu bebê, 13,19 entendemos que assim como para as outras 
formas de participação deve-se levar em conta seu desejo e suas possibilidades. É importante refletir que nessas situações de promoção da maternagem, da participação, nos cuidados assistenciais e no aleitamento materno, existe uma linha muito tênue entre o estímulo e a cobrança. 44

Desde o início ficou claro que os entrevistados confundiam "participar" com "assistir" quando questionados sobre os procedimentos em que a mãe participava. É claro que estar ao lado, tocar, já é participar, mas a pergunta referia-se a participação ativa/direta no cuidado. Dessa forma, "assistir" correspondeu a $73 \%$ dos profissionais que responderam no exame físico e a $51 \%$ nos procedimentos não invasivos. Um dado interessante foi que a maioria dos profissionais esclareceu deixar a critério da mãe permanecer ou não durante o procedimento, demonstrando um cuidado "novo" na prática dos profissionais de saúde.

Chamou-nos a atenção a dificuldade ainda presente nos profissionais em permitir a ida de um $\mathrm{RN}$ de risco ao colo. Apesar de 59\% responderem que depende de estabilidade clínica para ir ao colo materno pela primeira vez, apenas $30 \%$ responderam admitir esse contato com o bebê estabilizado, mas ainda entubado. Não há na literatura contra-indicação para que um RN, estável clinicamente, porém entubado, possa ir ao colo. Entendemos a necessidade de extrema cautela, assim como de equipe técnica disponível para tornar possível essa ação, porém chamou atenção o fato dos profissionais relutarem, francamente, em incorporar tal ação a sua prática.

Cerca de $99 \%$ dos entrevistados afirmaram estimular o contato pele-a-pele. Quanto ao momento em que o profissional estimula esse contato, $64 \%$ o fazem ainda na unidade de terapia intensiva (UTI) e $15 \%$ exclusivamente na unidade de cuidados inter- mediários (UI); no entanto, $26 \%$ dos profissionais confundem o ato de tocar, com contato pele-a-pele (RN ligeiramente vestido, em decúbito prono, na posição vertical, contra o peito do adulto).

\section{Considerações finais}

Ao final deste estudo, apesar de, em várias situações, ficar claro que muitos dos conceitos da atenção humanizada estão bastante difundidos e a priori o profissional os conhecer, eles ainda não foram plenamente incorporados em sua prática clínica. É grande a lacuna entre os conhecimentos e a aplicação prática das mudanças propostas pela AHRNBP-MC. Elas requerem mais do que a adoção de rotinas e o treinamento formal de alguns profissionais. É necessária a educação permanente em todos os níveis de formação dos profissionais de saúde que cuidam de RN.

A inadequação na estrutura das Unidades Neonatais em nosso país, encontrada também nos relatos dos profissionais das maternidades visitadas nesse estudo, certamente contribui para aumentar a dificuldade de implementação de uma prática comprometida com a singularidade de cada família. Por outro lado, embora a proposta brasileira inclua todo o período de internação do neonato, ficou evidente que os profissionais ainda relacionam basicamente a AHRNBP-MC à segunda etapa, a Unidade Canguru.

Por fim, é importante reiterar que a riqueza das propostas trazidas pela AHRNBP-MC justifica os esforços de todos os profissionais visando a superação dos desafios cotidianos. Nesse sentido, novos estudos que possam contribuir para a avaliação ampla da implantação e dos resultados do cuidado neonatal sob essa perspectiva certamente ajudarão na melhoria do cuidado neonatal em nosso país.

\section{Referências}

1. Gomes MASM. O Recém-Nascido de Alto Risco: Teoria e Prática do Cuidar. Organização da Assistência Perinatal no Brasil. In Moreira MEL, Lopes JMA, Carvalho M (org). Coleção Criança, Mulher e Saúde, Editora Fiocruz; 2002.p. 21-48.

2. Ministério da Saúde. Mortalidade perinatal e neonatal no Brasil. Brasília, DF, 1999.

3. Als H, Duffy FH, McAnulty GB, Rivkin MJ, Vajapeyan S, Mulkern RV et al. Early experience alters brain function and strutcture. Pediatrics 2004; 113: 846-57.

4. Levy GD, Woolston DJ, Browne JV. Mean Noise Amouns in Level II vs Level III. Neonatal Intensive Care Units. Neonatal Netw 2003; 22: 33-7.

5. Lansky S, França E, Leal MC. Mortalidade perinatal e evitabilidade: revisão de literatura. Rev Saúde Pública 2002; 36: 759-772.

6. Roqués V, Miranda J, Garrigues JV, Garcia A. Strategies to Improve the Neonatal Envitonment. In: Monduzzi Editore S.P.A. Medimond Inc. 2001: 5th World Congress of Perinatal Medicine, 2001. p. 1017-22.

7. Westrup B, Kleberg A, Stjernqvist K, Lagererantz. Newborn Individualized Developmental Care and Assessment Program (NIDCAP). In: Monduzzi Editore S.P.A. Medimond Inc. 2001:5th World Congress of Perinatal Medicine, 2001. p. 1029-36.

8. Hoath SB. The Skin as a Neurodevelopmental Interface. NeoReviews 2001; 2: e292-301. 
9. Klaus MH \& Kennel JH. Pais/Bebê: a formação do apego. Porto Alegre: Artes Médicas; 1993.

10. Silva OPV. Análise descritiva do desenvolvimento de recém-nascidos prematuros que participaram do programa Método Mãe-Canguru [dissertação]. São Paulo: Universidade Mackenzie; 2003.

11. Ministério da Saúde 2002. Atenção Humanizada ao RecémNascido de Baixo Peso - Método Mãe-Canguru - Manual Técnico.

12. Carvalho M, Gomes MA. A Mortalidade do prematuro extremo em nosso meio: realidade e desafios. J Pediatr (Rio J) 2005;81: S111-8.

13. Oliveira ND, Joaquim MCM. A Atenção Humanizada ao Recém-Nascido de Baixo Peso (Método Canguru) e a Amamentação. In Rego, editor. Aleitamento Materno. Rio de Janeiro: Editora Atheneu; 2002. p.401-8.

14. Kuzma-O'Reilly B, Mahe, Duenas ML et al. Evaluation, Development, and Implementation of Potentially Better Practices in Neonatal Intensive Care Nutrition. Pediatrics 2003; III (4):e461. Disponível em URL:http://www.pediatrics.org/cgi/content/full/iii/4/e461/. [2005 Fev 01].

15. Sharek PJ, Baker R, Litman F, Kaempf J, Burch K, Schwarz E et al. Evaluation and Development of Potentially Better Practices to Prevent Chronic Lung Disease and Reduce Lung Injury in Neonates. Pediatrics 2003; III (4):e426. Disponível em URL:http://www.pediatrics.org/cgi/content/ full/iii/4/e426/. [2005 Fev 01].

16. Kilbride HW, Wirtschafter DD, Powers RJ, Sheehan MB. Implementation of Evidence-Based Potentially Better Practices to Decrease Nosocomial Infections. Pediatrics 2003; III (4):e519. Disponível em URL:http://www.pediatrics.org/cgi/content/full/iii/4/e519/. [2005 Fev 01].

17. Jackson JK, Vellucci J, Johnson P, Kilbride HW. EvidenceBased Approach to Cange in Clinical Pratice: Introduction of Expanded Nasal Continuous Positive Airway Pressure Use in Intensive Care Nursery. Pediatrics 2003; III (4):e542. Disponível em URL:http://www.pediatrics.org/ cgi/content/ full/iii/4/e542/. [2005 Fev 01].

18. SMS/RJ - Secretaria municipal de Saúde. Gerência de Informação Epidemiológica. SINASC, 2002.

19. Braga NA, Morsch DS. Os Primeiros Dias na UTI. In: Moreira MEL, Braga NA e Morsch DS, organizadoras. Quando a Vida Começa Diferente - o bebê e sua família na UTI neonatal. Coleção Criança, Mulher e Saúde. Rio de Janeiro: Editora Fiocruz; 2003. p. 51-68.

20. Lamy ZC. Unidade Neonatal: um espaço de conflitos e negociações [Doutorado]. Rio de Janeiro: Instituto Fernandes Figueira-FIOCRUZ; 2000.

21. Mathelin C. O Sorriso de Gioconda: clínica psicanalítica com os bebês prematuros. Rio de Janeiro: Companhia de Freud; 1999.

22. Winnicott DW. Os Bebês e suas Mães. São Paulo: Martins Fontes; 1999.

23. Brazelton TB. Cramer BG. As Primeiras Relações. São Paulo: Martins Fontes; 1992.

24. Fabre-Grenet M. Os Meios de Comunicação do Prematuro. In: Busnel MC, organizadora. A Linguagem dos Bebês. Sabemos Escutá-los? São Paulo: Editora Escuta; 1997. p. 111-23.
25. Klaus MH, Kennell JH. Assistência aos pais. In: Alto risco em neonatologia. $4^{\mathrm{a}}$. Edição. Rio de Janeiro: Guanabara Koogan; 1995. p. 139-55.

26. Venancio SI, Almeida H. Método Mãe Canguru: aplicação no Brasil, evidências científicas e impacto sobre aleitamento materno. J Pediatr 2004; 80 (Supl 5):S173-80.

27. Carvalho, MLM. Participação dos pais no nascimento em maternidade pública: dificuldades institucionais e motivações dos casais. Cad Saúde Pública 2003; 19(Supl.2): S389-98.

28. Brazelton TB. O Desenvolvimento do Apego: uma família em formação. Porto Alegre: Artes Médicas, 1988.

29. Bowlby J. Apego. São Paulo: Martins Fontes; 1990. v.1.

30. Santoro WSJ, Santoro A. Reações Psicológicas e Processo Adaptativo de Pais de Recém-Nascidos Pré-Termo e de Muito Baixo Peso em Unidades de Terapia Intensiva Neonatal (UTIN). Rev Paul Pediatr 2002; 20: 95-101.

31. Padovani FHP, Linhares MBM, Carvalho AEV, Martinez FE, Duarte G. Indicadores Clínicos de Ansiedade e Depressão em Mães de Recém-Nascidos Pré-Termo de Muito Baixo Peso Comparados a Mães de Recém-Nascidos a Termo. Rev Paul Pediatr 2004; 22 (Suplemento 2004).

32. Preyde M, Ardal F. Effectiveness of a parent "buddy"program for mothers of very preterm infants in a neonatal intensive care unit. Can Med Assoc J 2003; 168: 969-73.

33. Saunders RP, Abraham MR, Crosby MJ, Thomas K, Edwards WH. Evaluation and Development of Potentially Better Practices for Improving Family-Centered Care in Neonatal Intensive Care Units. Pediatrics 2003; III (4):e437. Disponível em URL:http://www.pediatrics.org/ cgi/content/full/iii/4/e437/. [2005 Fev 01].

34. Moore KAC, Coker K, DuBuisson AB, Swett B, Edwards WH. Implementing Potentially Better Practices for Improving Family-Centered Care in Neonatal Intensive Care Units: Successes and Chalenges. Pediatrics 2003; III (4):e450-e460. Disponível em URL:http://www.pediatrics.org/cgi/content/full/iii/4/e450/. [2005 Fev 01].

35. Lamy ZC. Estudo das situações vivenciadas por pais de recém-nascidos internados em unidade de terapia intensiva neonatal [dissertação]. Rio de Janeiro: Instituto Fernandes Figueira-FIOCRUZ; 1995.

36. AAP. Committee on Enviroment Health Noise: a razard for the fetus and newborn. Pediatrics 1997; 100: 724-7.

37. Chermont AG, Guinsburg R, Balda RCX, Kopelman BI. O que os pediatras conhecem sobre avaliação e tratamento da dor no recém-nascido? J Pediatr 2003; 79: 265-72.

38. McGrath PJ, Unruh AM. The social context of neonatal pain. Clin Perinatol 2002; 29: 555-72.

39. Bhutta AT, Anand KJS. Vulnerability of the development brain Neuronal mechanisms. Clin Perinatol 2002; 29: 357 72.

40. AAP. Committee on Fetus and Newborn; Committee on Drugs; Section on Anestesiology; Section on Surgery; Canadian Paediatric Society. Fetus and Newborn Committee. Prevention and management of pain and stress in neonate. Pediatrics 2000; 105: 454-61. 
41. Porter FL, Wolf CM, Miller JP. Procedural Pain in Newborn Infants:The influence of Intensity and Development. Pediatrics 1999; 104: No.1:e13. Disponível em URL:http://www.pediatrics.org/cgi/content/ full/104/1/e13/. [2004 Out 10].

42. Lamy ZC. Metodologia Canguru: facilitando o encontro entre o bebê e sua família na UTI neonatal. In: Moreira MEL, Braga NA e Morsch DS, organizadoras. Quando a Vida Começa Diferente - o bebê e sua família na UTI neonatal. Coleção Criança, Mulher e Saúde. Rio de Janeiro: Editora Fiocruz; 2003. p.141-56

Recebido em 27 de julho de 2005

Versão final apresentada em 23 de outubro de 2006

Aprovado em 30 de outubro de 2006
43. Sabatés AL, Borba RIH. As informações recebidas pelos pais durante a hospitalização do filho. Rev Latinoam Enfermagem 2005; 13: 968-73.

44. Deslandes SF. Análise do discurso oficial sobre a humanização da assistência hospitalar. Ciênc Saúde Coletiva 9: 728 . 